\title{
ON TRAPEZOID INEQUALITY VIA A GRÜSS TYPE RESULT AND APPLICATIONS
}

\author{
S. S. DRAGOMIR AND A. MCANDREW
}

\begin{abstract}
In this paper, we point out a Grüss type inequality and apply it for special means (logarithmic mean, identric mean etc.. ) and in Numerical analysis in connection with the classical trapezoid formula.
\end{abstract}

\section{Introduction}

In 1935, G. Grüss (see for example [1, p. 296]), proved the following integral ineqality which gives an approximation for the integral of a product in terms of the product of integrals:

Theorem 1. Let $f, g:[a, b] \rightarrow \mathbb{R}$ be two integrable mappings so that $\varphi \leq f(x) \leq \Phi$ and $\gamma \leq g(x) \leq \Gamma$ for all $x \in[a, b]$, where $\varphi, \Phi, \gamma, \Gamma$ are real numbers. Then we have:

$$
\left|\frac{1}{b-a} \int_{a}^{b} f(x) g(x) d x-\frac{1}{b-a} \int_{a}^{b} f(x) d x \cdot \frac{1}{b-a} \int_{a}^{b} g(x) d x\right| \leq \frac{1}{4}(\Phi-\varphi)(\Gamma-\gamma)
$$

and the inequality is sharp, in the sense that the constant $\frac{1}{4}$ can not be replaced by a smaller one.

For a simple proof of this fact as well as for extensions, generalizations, discrete variants and other associated material, see [1, p. 296], and the papers [2]-[7] where further references are given.

In this paper, we point out a different Grüss type inequality and apply it for special means (logarithmic mean, identric mean, etc.. ) and in Numerical Analysis in connection with the classical trapezoid formula.

\section{A Grüss' Type Inequality}

We start with the following result of Grüss' type.

Received May 27, 1999; revised February 18, 2000.

2000 Mathematics Subject Classification. Primary 26D15, 26D20, Secondary 41A05.

Key words and phrases. Grüss Inequality, Trapezoid Inequality. 
Theorem 2. Let $f, g:[a, b] \rightarrow \mathbb{R}$ be two integrable mappings. Then we have the following Grüss' type inequality.

$$
\begin{aligned}
& \left|\frac{1}{b-a} \int_{a}^{b} f(x) g(x) d x-\frac{1}{b-a} \int_{a}^{b} f(x) d x \cdot \frac{1}{b-a} \int_{a}^{b} g(x) d x\right| \\
\leq & \frac{1}{b-a} \int_{a}^{b}\left|\left(f(x)-\frac{1}{b-a} \int_{a}^{b} f(y) d y\right) \cdot\left(g(x)-\frac{1}{b-a} \int_{a}^{b} g(y) d y\right)\right| d x .
\end{aligned}
$$

The inequality (2.1) is sharp.

Proof. First of all, let us observe that

$$
\begin{aligned}
I:= & \frac{1}{b-a} \int_{a}^{b}\left(f(x)-\frac{1}{b-a} \int_{a}^{b} f(y) d y\right) \cdot\left(g(x)-\frac{1}{b-a} \int_{a}^{b} g(y) d y\right) d x \\
= & \frac{1}{b-a} \int_{a}^{b}\left(f(x) g(x)-g(x) \cdot \frac{1}{b-a} \int_{a}^{b} f(y) d y-f(x) \cdot \frac{1}{b-a} \int_{a}^{b} g(y) d y\right. \\
& \left.+\frac{1}{b-a} \int_{a}^{b} f(y) d y \cdot \frac{1}{b-a} \int_{a}^{b} g(y) d y\right) d x \\
= & \frac{1}{b-a} \int_{a}^{b} f(x) g(x) d x-\frac{1}{b-a} \int_{a}^{b} g(x) d x \cdot \frac{1}{b-a} \int_{a}^{b} f(y) d y \\
& -\frac{1}{b-a} \int_{a}^{b} f(x) d x \cdot \frac{1}{b-a} \int_{a}^{b} g(y) d y+\frac{1}{b-a} \int_{a}^{b} f(y) d y \cdot \frac{1}{b-a} \int_{a}^{b} g(y) d y \\
= & \frac{1}{b-a} \int_{a}^{b} f(x) g(x) d x-\frac{1}{b-a} \int_{a}^{b} g(x) d x \cdot \frac{1}{b-a} \int_{a}^{b} f(x) d x .
\end{aligned}
$$

On the other hand, by the use of modulus properties, we have

$$
|I| \leq \frac{1}{b-a} \int_{a}^{b}\left|\left(f(x)-\frac{1}{b-a} \int_{a}^{b} f(y) d y\right) \cdot\left(g(x)-\frac{1}{b-a} \int_{a}^{b} g(y) d y\right)\right| d x
$$

and the inequality $(2.1)$ is proved.

Choosing $f(x)=g(x)=\operatorname{sgn}\left(x-\frac{a+b}{2}\right)$, the equality is satisfied in (2.1).

The following corollaries follow immediately:

Corollary 1. Let $f:[a, b] \rightarrow \mathbb{R}$ be a differentiable mapping on $(a, b)$ having the first derivative $f^{\prime}:(a, b) \rightarrow \mathbb{R}$ bounded on $(a, b)$. Then we have the inequality:

$$
\left|\frac{f(a)+f(b)}{2}-\frac{1}{b-a} \int_{a}^{b} f(x) d x\right| \leq \frac{b-a}{4} \sup _{x \in(a, b)}\left|f^{\prime}(x)-\frac{f(b)-f(a)}{b-a}\right| .
$$

Proof. A simple integration by parts gives that:

$$
\frac{f(a)+f(b)}{2}(b-a)-\int_{a}^{b} f(x) d x=\int_{a}^{b}\left(x-\frac{a+b}{2}\right) f^{\prime}(x) d x .
$$


Applying the inequality (2.1) we find that:

$$
\begin{aligned}
& \left|\int_{a}^{b} \frac{1}{b-a}\left(x-\frac{a+b}{2}\right) f^{\prime}(x) d x-\frac{1}{b-a} \int_{a}^{b}\left(x-\frac{a+b}{2}\right) d x \cdot \frac{1}{b-a} \int_{a}^{b} f^{\prime}(x) d x\right| \\
\leq & \frac{1}{b-a} \int_{a}^{b}\left|\left(x-\frac{a+b}{2}-\frac{1}{b-a} \int_{a}^{b}\left(y-\frac{a+b}{2}\right) d y\right) \cdot\left(f^{\prime}(x)-\frac{1}{b-a} \int_{a}^{b} f^{\prime}(y) d y\right)\right| d x .
\end{aligned}
$$

As

$$
\int_{a}^{b}\left(x-\frac{a+b}{2}\right) d x=0
$$

we obtain

$$
\begin{aligned}
\left|\int_{a}^{b}\left(x-\frac{a+b}{2}\right) f^{\prime}(x) d x\right| & \leq \int_{a}^{b}\left|\left(x-\frac{a+b}{2}\right)\left(f^{\prime}(x)-\frac{f(b)-f(a)}{b-a}\right)\right| d x \\
& \leq \sup _{x \in(a, b)}\left|f^{\prime}(x)-\frac{f(b)-f(a)}{b-a}\right| \int_{a}^{b}\left|x-\frac{a+b}{2}\right| d x \\
& =\frac{(b-a)^{2}}{4} \sup _{x \in(a, b)}\left|f^{\prime}(x)-\frac{f(b)-f(a)}{b-a}\right|
\end{aligned}
$$

Now, using the equality (2.3), the inequality (2.4) becomes the desired result (2.2).

Corollary 2. Suppose $p, q>1$ satisfy $\frac{1}{p}+\frac{1}{q}=1$. Let $f:[a, b] \rightarrow \mathbb{R}$ be a differentiable mapping on $(a, b)$ having the first derivative $f^{\prime}:(a, b) \rightarrow \mathbb{R}$ being q-integrable on $(a, b)$. Then we have the inequality:

$$
\left|\frac{f(a)+f(b)}{2}-\frac{1}{b-a} \int_{a}^{b} f(x) d x\right| \leq \frac{1}{2}\left(\frac{b-a}{p+1}\right)^{\frac{1}{p}}\left(\int_{a}^{b}\left|f^{\prime}(x)-\frac{f(b)-f(a)}{b-a}\right|^{q} d x\right)^{\frac{1}{q}} .
$$

Proof. Using Hölder's inequality, we have that:

$$
\begin{aligned}
& \int_{a}^{b}\left|\left(x-\frac{a+b}{2}\right)\left(f^{\prime}(x)-\frac{f(b)-f(a)}{b-a}\right)\right| d x \\
\leq & \left(\int_{a}^{b}\left|x-\frac{a+b}{2}\right|^{p} d x\right)^{\frac{1}{p}}\left(\int_{a}^{b}\left|f^{\prime}(x)-\frac{f(b)-f(a)}{b-a}\right|^{q} d x\right)^{\frac{1}{q}} \\
= & \frac{(b-a)^{\frac{1}{p}+1}}{2(p+1)^{\frac{1}{p}}}\left(\int_{a}^{b}\left|f^{\prime}(x)-\frac{f(b)-f(a)}{b-a}\right|^{q} d x\right)^{\frac{1}{q}} .
\end{aligned}
$$

Now, using the first part of (2.4) and the identity (2.3), we obtain the desired result $(2.5)$.

The following result also holds. 
Corollary 3. Let $f:[a, b] \rightarrow \mathbb{R}$ be a differentiable mapping on $(a, b)$ and suppose that $f^{\prime}:(a, b) \rightarrow \mathbb{R}$ is integrable on $(a, b)$. Then we have the inequality:

$$
\left|\frac{f(a)+f(b)}{2}-\frac{1}{b-a} \int_{a}^{b} f(x) d x\right| \leq \frac{1}{2} \int_{a}^{b}\left|f^{\prime}(x)-\frac{f(b)-f(a)}{b-a}\right| d x .
$$

Proof. We have

$$
\begin{aligned}
& \int_{a}^{b}\left|\left(x-\frac{a+b}{2}\right)\left(f^{\prime}(x)-\frac{f(b)-f(a)}{b-a}\right)\right| d x \\
\leq & \sup _{x \in(a, b)}\left|x-\frac{a+b}{2}\right| \cdot \int_{a}^{b}\left|f^{\prime}(x)-\frac{f(b)-f(a)}{b-a}\right| d x \\
= & \frac{b-a}{2} \int_{a}^{b}\left|f^{\prime}(x)-\frac{f(b)-f(a)}{b-a}\right| d x .
\end{aligned}
$$

Using the first part of (2.4) and the identity (2.3), we obtain the desired result (2.6).

\section{Applications for Some Special Means}

In this section we shall refer to the following special means

(a) The arithmetic mean

$$
A=A(a, b):=\frac{a+b}{2}, \quad a, b \geq 0
$$

(b) The geometric mean

$$
G=G(a, b):=\sqrt{a b}, \quad a, b \geq 0
$$

(c) The harmonic mean

$$
H=H(a, b):=\frac{2}{\frac{1}{a}+\frac{1}{b}}, \quad a, b>0
$$

(d) The logarithmic mean

$$
L=L(a, b):=\left\{\begin{array}{l}
a \text { if } b=a \\
\frac{b-a}{\ln b-\ln a} \text { if } b \neq a, \quad a, b>0
\end{array}\right.
$$

(e) The identric mean

$$
I=I(a, b):=\left\{\begin{array}{l}
a \text { if } a=b \\
\frac{1}{e}\left(\frac{b^{b}}{a^{a}}\right)^{\frac{1}{b-a}} \text { if } b \neq a, \quad a, b>0
\end{array}\right.
$$

(f) The p-logarithmic mean

$$
L_{p}=L_{p}(a, b):=\left\{\begin{array}{l}
a \text { if } b=a \\
{\left[\frac{b^{p+1}-a^{p+1}}{(p+1)(b-a)}\right]^{\frac{1}{p}} \text { if } b \neq a, \quad a, b>0}
\end{array}\right.
$$


where $p \in \mathbb{R} \backslash\{-1,0\}$.

It is well known that

$$
H \leq G \leq L \leq I \leq A
$$

and the mapping $L_{p}$ is monotonically increasing in $p \in \mathbb{R}$ with $L_{0}:=I$ and $L_{-1}=: L$.

I. Now, let us consider inequality $(2.2)$, with $f:(0, \infty) \rightarrow \mathbb{R}$ defined by $f(x)=x^{r}, r \in$ $\mathbb{R} \backslash\{0,-1\}$. Then for $0<a<b$, we have

$$
\left|\frac{f(a)+f(b)}{2}-\frac{1}{b-a} \int_{a}^{b} f(x) d x\right| \leq \frac{b-a}{4} \sup _{x \in(a, b)}\left|f^{\prime}(x)-\frac{f(b)-f(a)}{b-a}\right|,
$$

where $f$ is as in Corollary 1 .

1. Consider the mapping $f:(0, \infty) \rightarrow \mathbb{R}, f(x)=x^{r}, r \in \mathbb{R} \backslash\{-1,0\}$. Then for $0<a<b$, we have

$$
\begin{aligned}
\frac{f(a)+f(b)}{2} & =A\left(a^{r}, b^{r}\right), \\
\frac{1}{b-a} \int_{a}^{b} f(x) d x & =L_{r}^{r}(a, b), \\
f^{\prime}(x)-\frac{f(b)-f(a)}{b-a} & =r x^{r-1}-r L_{r-1}^{r-1}=r\left(x^{r-1}-L_{r-1}^{r-1}\right),
\end{aligned}
$$

and by the inequality 3.2 we obtain:

$$
\left|A\left(a^{r}, b^{r}\right)-L_{r}^{r}(a, b)\right| \leq \frac{|r|(b-a)}{4} \sup _{x \in(a, b)}\left|x^{r-1}-L_{r-1}^{r-1}\right| .
$$

2. Consider the mapping $f:(0, \infty) \rightarrow \mathbb{R}, f(x)=\frac{1}{x}$. Then for $0<a<b$, we have

$$
\begin{aligned}
\frac{f(a)+f(b)}{2} & =\frac{A(a, b)}{G^{2}(a, b)}, \\
\frac{1}{b-a} \int_{a}^{b} f(x) d x & =\frac{1}{L(a, b)}, \\
f^{\prime}(x)-\frac{f(b)-f(a)}{b-a} & =-\frac{1}{x^{2}}+\frac{1}{a b}, \\
\sup _{x \in(a, b)}\left|f^{\prime}(x)-\frac{f(b)-f(a)}{b-a}\right| & =\frac{(b-a)}{a^{2} b},
\end{aligned}
$$

and by the inequality (3.2) we obtain

$$
\left|\frac{A}{G^{2}}-\frac{1}{L}\right| \leq \frac{(b-a)^{2}}{4 a G^{2}}
$$

which is equivalent to

$$
0 \leq L A-G^{2} \leq \frac{(b-a)^{2}}{4 a} L
$$


3. Consider the mapping $f:(0, \infty) \rightarrow \mathbb{R}, f(x)=\ln x$. Then for $0<a<b$, we have

$$
\begin{aligned}
\frac{f(a)+f(b)}{2} & =\ln G, \\
\frac{1}{b-a} \int_{a}^{b} f(x) d x & =\ln I, \\
f^{\prime}(x)-\frac{f(b)-f(a)}{b-a} & =\frac{1}{x}-\frac{1}{L}, \\
\sup _{x \in(a, b)}\left|f^{\prime}(x)-\frac{f(b)-f(a)}{b-a}\right| & =\frac{1}{a}-\frac{1}{L}=\frac{L-a}{a L}
\end{aligned}
$$

and by the inequality (3.2) we obtain

$$
|\ln G-\ln I| \leq \frac{(b-a)}{4} \cdot\left(\frac{L-a}{a L}\right)
$$

which is equivalent to:

$$
1 \leq \frac{I}{G} \leq \exp \left(\frac{(b-a)}{4} \cdot\left(\frac{L-a}{a L}\right)\right)
$$

II. Now, let us consider inequality (2.6) with $f:(0, \infty) \rightarrow \mathbb{R}$ defined by $f(x)=x^{r}$, $r \in \mathbb{R} \backslash\{0,-1\}$. Then for $0<a<b$ we have

$$
\left|\frac{f(a)+f(b)}{2}-\frac{1}{b-a} \int_{a}^{b} f(x) d x\right| \leq \frac{1}{2} \int_{a}^{b}\left|f^{\prime}(x)-\frac{f(b)-f(a)}{b-a}\right| d x .
$$

1. Consider the mapping $f:(0, \infty) \rightarrow \mathbb{R} f(x)=x^{r}, r \in \mathbb{R} \backslash\{0,-1\}$ and $0<a<b$. Then

$$
\int_{a}^{b}\left|f^{\prime}(x)-\frac{f(b)-f(a)}{b-a}\right| d x=|r| \int_{a}^{b}\left|x^{r-1}-L_{r-1}^{r-1}\right| d x .
$$

For simplicity, let assume that $r>1$. Then

$$
\begin{aligned}
\int_{a}^{b}\left|x^{r-1}-L_{r-1}^{r-1}\right| d x & =\int_{a}^{L_{r-1}}\left(L_{r-1}^{r-1}-x^{r-1}\right) d x+\int_{L_{r-1}}^{b}\left(x^{r-1}-L_{r-1}^{r-1}\right) d x \\
& =L_{r-1}^{r-1}\left(L_{r-1}-a\right)-\left.\frac{x^{r}}{r}\right|_{a} ^{L_{r-1}}+\left.\frac{x^{r}}{r}\right|_{L_{r-1}} ^{b}-\left(b-L_{r-1}\right) L_{r-1}^{r-1} \\
& =L_{r-1}^{r-1}-a L_{r-1}^{r-1}-\frac{L_{r-1}^{r}-a^{r}}{r}+\frac{b^{r}-L_{r-1}^{r}}{r}-\left(b-L_{r-1}\right) L_{r-1}^{r-1} \\
& =\frac{b^{r}+a^{r}}{r}-L_{r-1}^{r-1}(a+b)-\frac{2 L_{r-1}^{r}}{r}+2 L_{r-1}^{r} \\
& =\frac{2}{r}\left[A\left(a^{r}, b^{r}\right)-r L_{r-1}^{r-1} A+L_{r-1}^{r}(r-1)\right]
\end{aligned}
$$


and by the inequality (3.6) we obtain

$$
0 \leq A\left(a^{r}, b^{r}\right)-L_{r}^{r}(a, b) \leq\left[A\left(a^{r}, b^{r}\right)-r L_{r-1}^{r-1} A+L_{r-1}^{r}(r-1)\right]
$$

or

$$
r L_{r-1}^{r-1} A \leq L_{r}^{r}(a, b)+(r-1) L_{r-1}^{r} .
$$

Similar results can be obtained for $r \leq 1, r \neq 0,-1$.

We shall omit the details.

2. Consider the mapping $f(a, b) \rightarrow \mathbb{R}, f(x)=\frac{1}{x}$. Then for $0<a<b$ we have:

$$
\begin{aligned}
& \int_{a}^{b}\left|\frac{-1}{x^{2}}+\frac{1}{a b}\right| d x=\frac{1}{a b} \int_{a}^{b}\left|1-\frac{a b}{x^{2}}\right| d x \\
= & \frac{1}{a b}\left[\int_{a}^{\sqrt{a} b}\left(\frac{a b}{x^{2}}-1\right) d x+\int_{\sqrt{a b}}^{b}\left(1-\frac{a b}{x^{2}}\right) d x\right]=\frac{4}{G^{2}(A-G)}
\end{aligned}
$$

and by inequality (3.6) we obtain:

$$
\left|\frac{A}{G^{2}}-\frac{1}{L}\right| \leq \frac{2(A-G)}{G^{2}}
$$

i.e.,

$$
0 \leq A L-G^{2} \leq 2 L(A-G)
$$

or equivalenty:

$$
2 L G \leq G^{2}+A L
$$

which is a very interesting inequality amongst $A, L$ and $G$.

3. Consider the mapping $f:(a, b) \rightarrow \mathbb{R}, f(x)=\ln x$. Then for $0<a<b$, we have:

$$
\begin{aligned}
\frac{1}{2} \int_{a}^{b}\left|\frac{1}{x}-\frac{1}{L}\right| d x & =\int_{a}^{b} \frac{|x-L|}{x L} d x=\int_{a}^{L}\left(\frac{1}{x}-\frac{1}{L}\right) d x+\int_{L}^{b}\left(\frac{1}{L}-\frac{1}{x}\right) d x \\
& =\frac{A-L}{L}+\ln L-\ln G
\end{aligned}
$$

and then by the inequality (3.6) we obtain.

$$
|\ln G-\ln I| \leq \ln L-\ln G+\frac{A-L}{L}=\ln \left[\left(\frac{L}{G}\right) \exp \left(\frac{A-L}{L}\right)\right]
$$

i.e.,

$$
1 \leq \frac{I}{G} \leq \frac{L}{G} \exp \left(\frac{A-L}{L}\right)
$$

which implies

$$
\frac{G}{L} \leq \frac{I}{L} \leq \exp \left(\frac{A-L}{L}\right)
$$




\section{Applications for the Trapezoid Formula}

In this section we shall assume that $f: I \subseteq \mathbb{R} \rightarrow \mathbb{R}$ is a differentiable mapping whose derivative satisfies the following condition:

$$
\left|f(b)-f(a)-(b-a) f^{\prime}(x)\right| \leq \Omega(b-a)^{2}, \quad \Omega>0
$$

for all $a, b \in I$ and $x$ between $a$ and $b$.

If $f^{\prime}$ is $M$-lipschitzian, i.e.,

$$
\left|f^{\prime}(u)-f^{\prime}(v)\right| \leq M|u-v|, \quad M>0
$$

then

$$
\begin{aligned}
\left|f(b)-f(a)-(b-a) f^{\prime}(x)\right| & =\left|f^{\prime}(c)-f^{\prime}(x)\right||b-a| \\
& \leq M|b-a||c-x| \leq M(b-a)^{2}
\end{aligned}
$$

where $c$ is between $a$ and $b$, too. Consequently, the mappings having the first derivative lipschitzian satisfy the condition (4.1).

The following trapezoid formula holds.

Theorem 3. Let $f:[a, b] \rightarrow \mathbb{R}$ be differentiable mapping on $(a, b)$ whose derivative $f^{\prime}:(a, b) \rightarrow \mathbb{R}$ satisfies the above conditon (4.1) on $(a, b)$. If $I_{h}: a=x_{0}<x_{1}<\cdots<$ $x_{n-1}<x_{n}=b$ is a partition of $[a, b]$ and $h_{i}=x_{i+1}-x_{i}, i=0, \ldots, n-1$, then we have:

$$
\int_{a}^{b} f(t) d t=A_{T, I_{h}}(f)+R_{T, I_{h}}(f)
$$

where

$$
A_{T, I_{h}}(f)=\sum_{i=0}^{n-1} \frac{f\left(x_{i}\right)+f\left(x_{i+1}\right)}{2} h_{i}
$$

and the remainder $R_{T, I_{h}}(f)$ satisfies the estimation:

$$
\left|R_{T, I_{h}}(f)\right| \leq \frac{\Omega}{4} \sum_{i=0}^{n-1} h_{i}^{3} .
$$

Proof. Corollary 1 applied to the interval $\left[x_{i}, x_{i+1}\right]$ gives:

$$
\begin{aligned}
& \left|\left(x_{i+1}-x_{i}\right) \frac{f\left(x_{i}\right)+f\left(x_{i+1}\right)}{2}-\int_{x_{i}}^{x_{i+1}} f(t) d t\right| \\
\leq & \frac{\left(x_{i+1}-x_{i}\right)^{2}}{4} \sup _{x \in\left(x_{i}, x_{i+1}\right)}\left|f^{\prime}(x)-\frac{f\left(x_{i+1}\right)-f\left(x_{i}\right)}{x_{i+1}-x_{i}}\right| \\
\leq & \frac{\Omega\left(x_{i+1}-x_{i}\right)^{3}}{4}
\end{aligned}
$$


i.e.,

$$
\left|\frac{f\left(x_{i}\right)+f\left(x_{i+1}\right)}{2} h_{i}-\int_{x_{i}}^{x_{i+1}} f(t) d t\right| \leq \frac{\Omega h_{i}^{3}}{4} \text { for all } i=0, \ldots, n-1 .
$$

Summing the above inequality and using the generalized triangle inequality, we obtain the approximation (4.2) and the remainder satisfies the estimation (4.4).

Remark 1. We have obtained in this way a trapezoid formula for a class larger than the class $C^{2}[a, b]$, for which the usual trpezoid formula holds with the remainder term satisfiying

$$
\left|R_{T, I_{h}}(f)\right| \leq \frac{\left\|f^{\prime \prime}\right\|_{\infty}}{12} \sum_{i=0}^{n-1} h_{i}^{3}
$$

where $\left\|f^{\prime \prime}\right\|_{\infty}=\sup _{t \in(a, b)}\left|f^{\prime \prime}(t)\right|<\infty$.

\section{References}

[1] D. S. Mitrinović, J. E. Pečarić and A. M. Fink, Classical and New Inequalities in Analysis, Kluwer Academic publishers, Dordrecht, 1993.

[2] S. S. Dragomir, Some integral inequalities of Grüss type, accepted in Indian Journal of Pure and Appl. Math..

[3] S. S. Dragomir, Grüss inequality in inner product spaces, Austral. Math. Soc., 26(1999), 66-70.

[4] S. S. Dragomir, New estimation of the remainder in Taylor's formula using Grüss' type inequalities and appliciations, Mathematical Inequalities and Applications, 2(1999), 183193.

[5] S. S. Dragomir and I. Fedotov, An inequality of Grüss type for Riemann-Stieltjes integral and applications for special means, Tamkang Journal of Mathematics., 29(1998), 287-292.

[6] I. Fedotov and S. S. Dragomir, Another approach to quadrature methods, Indian Journal of Pure and Applied Mathematics, 30(1999), 763-775.

[7] S. S. Dragomir, A Grüss type integral inequality for mappings of $r$-Holder's type and applications for trapezoid formula, Tamkang J. of Math., 31(2000),93-97.

School of Communications and Informatics, Victoria University of Technology, PO Box 14428, MC Melbourne City, Victoria 8001, Australia.

E-mail: \{sever, amca\}@matilda.vu.edu.au

URL: http://matilda.vu.edu.au/ ${ }^{\sim}$ rgmia/dragomirweb.html 